\title{
Direct Interaction Force and Adsorption Behavior of Fibrinogen on Well-Characterized Polymer Brush Surfaces
}

\author{
Sho Sakata*, Yuuki Inoue, and Kazuhiko Ishihara \\ Department of Materials Engineering, School of Engineering, The University of Tokyo \\ Bunkyo-ku, Tokyo 113-8656 Japan \\ Fax: 81-3-5841-8647, e-mail: sakata@mpc.t.u-tokyo.ac.jp
}

\begin{abstract}
Adsorption behavior of fibrinogen was examined in terms of the direct interaction force operating between fibrinogen and surfaces. As model surfaces, well-characterized polymer brush surfaces were systematically prepared by surface-initiated atom transfer radical polymerization method using zwitterionic, cationic, anionic, and hydrophobic monomers. The direct interaction forces between fibrinogen and the polymer brush surfaces were quantitatively evaluated by the force-versus-distance curve measurement of atomic force microscopy using fibrinogen-immobilized probe. Besides, the amount of fibrinogen adsorbed on these polymer brush surfaces was quantified by surface plasmon resonance measurement. The cationic, anionic, and hydrophobic polymer brush surfaces strongly interacted with fibrinogen, and a large amount of fibrinogen adsorbed on them. On the other hand, in the case of the zwitterionic polymer brush surface, direct interaction force with fibrinogen was weak, and fibrinogen adsorption was dramatically suppressed. It was quantitatively indicated that the strength of direct interaction force operating between fibrinogen and surfaces would influence on fibrinogen adsorption on surfaces.

Key words: Polymer brush surface, Electrostatic interaction, Hydrophobic interaction, Atomic force microscopy, Protein adsorption, Fibrinogen
\end{abstract}

\section{INTRODUCTION}

Protein adsorption on the materials surfaces is one of the most fundamental issues in the field of biomaterials science because protein adsorption is the initial event of biological reactions induced at the biointerface [1]. Fibrinogen is one of the most common proteins comprised in blood plasma, and related to unfavorable biological responses such as blood coagulation and clot formation [2]. Therefore, fibrinogen adsorption must be suppressed to a maximum extent in order to eliminate non-specific biological responses on the surface of blood-contacting devices. Generally, protein adsorption proceeds in a very complex manner, and various molecular interaction forces are operating on each step [3-4]. However, the relationship between such molecular interaction forces and protein adsorption behavior has not been clarified. This study aimed to quantitatively understand protein adsorption phenomena based on analysis of molecular interaction forces.

In our previous study, systematic polymer brush surfaces were fabricated by surface-initiated atom transfer radical polymerization (SI-ATRP) method using zwitterionic, cationic, anionic, and hydrophobic monomers as model surfaces [5]. Surface force analysis by atomic force microscopy (AFM) revealed that the cationic and anionic polymer brush surfaces generated only electrostatic interaction force, and the hydrophobic polymer brush surface generated only hydrophobic interaction force, whereas the zwitterionic polymer brush surface exhibited no interaction force. In the present study, the same polymer brush surface systems were utilized to investigate the fibrinogen adsorption behavior based on the interaction forces. As a preliminary evaluation to understand fibrinogen adsorption process, the direct interaction force between fibrinogen and polymer brush surfaces was quantitatively evaluated by the force-versus-distance $(f-d)$ curve measurements of AFM using the fibrinogen-immobilized probe (Fig. 1), and adsorption behavior of fibrinogen on the surfaces was evaluated in terms of whole mass. The relationship between the direct interaction force with fibrinogen, and the amount of adsorbed fibrinogen on the polymer brush layers was investigated.

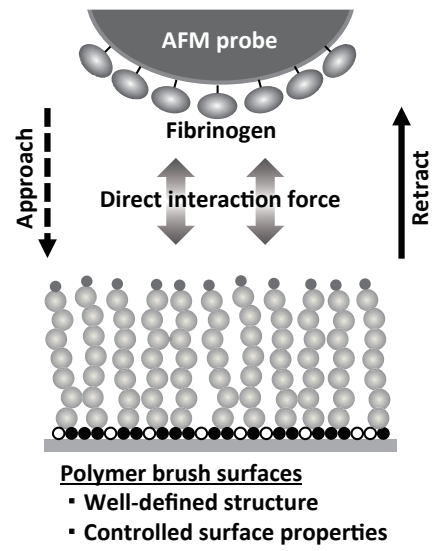

Fig. 1. Schematic representation of the measurement of the direct interaction force between fibrinogen and polymer brush layers by AFM. 


\section{MATERIALS AND METHODS}

\subsection{Materials}

2-Methacryloyloxyethyl phosphorylcholine (MPC) was purchased from NOF Corp. (Tokyo, Japan), which was synthesized and purified according to a previously reported method [6]. 2-Trimethylammoniumethyl methacrylate chloride (TMAEMA) and 3-sulfopropyl methacrylate potassium salt (SPMA) were purchased from Tokyo Chemical Industry Co., Ltd. (Tokyo, Japan). $n$-Butyl methacrylate (BMA) was purchased from Kanto Chemical Co., Inc. (Tokyo, Japan). Copper(I) bromide $(\mathrm{CuBr})$ and 2,2'-bipyridyl (Bpy) were purchased from Wako Pure Chemical Industries Ltd. (Osaka, Japan). Ethyl-2-bromoisobutyrate (EBIB), 4,4'-dinonyl-2,2'bipyridyl (DNbpy), and fibrinogen from bovine plasma were purchased from Sigma-Aldrich Co. (St. Louis, MO, USA). Silicon wafers were purchased from Furuuchi Chemical Corp. (Tokyo, Japan); their surface were coated with $\sim 10$-nm-thick $\mathrm{SiO}_{2}$ layers.

\subsection{Preparation of polymer brush layers}

Polymer brush layers were prepared on the initiator-immobilized substrates by SI-ATRP using MPC, TMAEMA, SPMA, and BMA according to a previously reported procedure [5]. Briefly, a surface-immobilizing initiator, (10-(2-bromo-2-methyl)propyonyloxy)decyltrichlorosilane (BrC10TCS), was synthesized and immobilized on the silicon substrates as previously described [7]. Specific amounts of $\mathrm{CuBr}$, Bpy, and the monomers were dissolved in the degassed solvents. Potassium chloride of the same concentration as SPMA was added to the SPMA solution to enhance the solubility of SPMA. DNbpy was used as the ligand instead of Bpy for the polymerization of BMA. Then, the BrC10TCS-immobilized substrates and EBIB, as the free initiator, were simultaneously added into the solution to initiate SI-ATRP. Polymerization was performed with stirring at $20^{\circ} \mathrm{C}$ for $24 \mathrm{~h}$. The target degree of polymerization ([monomer]/[free initiator] ratio in feed) was set at 100 . Fig. 2 shows the chemical structures of the polymer brush layers, and Table I summarizes the physicochemical properties of the polymer brush layers.

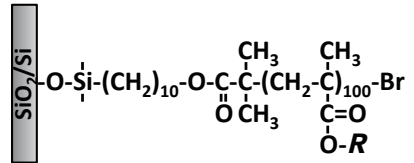

$$
R= \begin{cases}\left(\mathrm{CH}_{2}\right)_{2} \mathrm{OP} \underset{1}{\mathrm{O} O}\left(\mathrm{CH}_{2}\right)_{2} \mathrm{~N}^{+}\left(\mathrm{CH}_{3}\right)_{3} & \text { Poly(MPC) } \\ \mathrm{O}^{-} & \\ \left(\mathrm{CH}_{2}\right)_{2} \mathrm{~N}^{+}\left(\mathrm{CH}_{3}\right)_{3} \cdot \mathrm{Cl}^{-} & \text {Poly(TMAEMA) } \\ \left(\mathrm{CH}_{2}\right)_{3} \mathrm{SO}_{3}^{-} \cdot \mathrm{K}^{+} & \text {Poly(SPMA) } \\ \left(\mathrm{CH}_{2}\right)_{3} \mathrm{CH}_{3} & \text { Poly(BMA) }\end{cases}
$$

Fig. 2. Chemical structures of the polymer brush layers.

\subsection{Interaction force measurement}

The direct interaction force between fibrinogen and the polymer brush surfaces was quantitatively evaluated by the $f-d$ curve measurements using an AFM equipped with a liquid cell (Nanoscope IIIa; Bruker AXS K.K., Kanagawa, Japan) according to a previously reported method [8]. Briefly, 3-nm-thick chromium and sequential 27-nm-thick gold were sputtered on the surface of AFM probe (OTR8; nominal spring constant $0.15 \mathrm{~N} / \mathrm{m}$, Bruker AXS K.K.). The gold-sputtered probe was then immersed in a $1.0 \mathrm{mmol} / \mathrm{L}$ solution of 11-mercaptoundecanoic acid in ethanol for $24 \mathrm{~h}$ to form a carboxyl-group-terminated self-assembled monolayer on the probe. The carboxyl groups were activated by immersion in an aqueous solution containing $\mathrm{N}$-hydroxysuccinimide $(50 \mathrm{mmol} / \mathrm{L})$ and 1-ethyl-3(3-dimethylaminopropyl)carbodiimide hydrochloride (100 $\mathrm{mmol} / \mathrm{L})$. After immersion for $30 \mathrm{~min}$, the probe was rinsed with pure water and immediately immersed in a phosphate-buffered saline (PBS; $\mathrm{pH} 7.4$, ionic strength $=150 \mathrm{mmol} / \mathrm{L})$ solution of fibrinogen $(0.10$ $\mathrm{mg} / \mathrm{mL}$ ) for $1 \mathrm{~h}$. The direct interaction force between fibrinogen and the polymer brush surfaces was evaluated from the approaching and retracting trace of the $f-d$ curve using the fibrinogen-immobilized probes. The shift in the deflection value of the retracting trace from the bottom of the retrace line corresponds to the interaction force between fibrinogen and surfaces. For each sample, more than 100 approaching/retracting $f$ - $d$ curves were collected and the average value was defined as the interaction force.

\subsection{Protein adsorption measurement}

The amount of fibrinogen adsorbed on the polymer brush surfaces in PBS at $37^{\circ} \mathrm{C}$ was quantified by surface plasmon resonance (SPR) (SPR-670M; Moritex Co., Tokyo, Japan) measurement. The polymer brush layers were prepared on SPR sensor chips by SI-ATRP using 11-(2-bromo-2-methylpropyonyloxy)undecylmercaptan (BUM) as the surface-immobilizing initiator for the thin gold layer on the substrate [9]. A peristaltic pump (Tokyo Rika Kikai Co., Ltd., Tokyo, Japan) was used to flow the buffer solution or protein solution thorough the SPR sensor surfaces at a rate of $500 \mu \mathrm{L} / \mathrm{min}$. First, a stable baseline signal was established by flowing buffer solution for $10 \mathrm{~min}$. Then, $1.0 \mathrm{mg} / \mathrm{mL}$ fibrinogen solution was flowed for $30 \mathrm{~min}$, followed by buffer solution for $10 \mathrm{~min}$ to replace protein solution, wash off the weakly adsorbed proteins from the surface, and re-establish the baseline. The amount of adsorbed fibrinogen, $\Gamma_{\mathrm{SPR}}\left(\mathrm{ng} / \mathrm{cm}^{2}\right)$, was estimated using the following relationship [10]:

$$
\Gamma_{\mathrm{SPR}}=500 \times \Delta R_{\mathrm{deg}}
$$

where $\Delta R_{\operatorname{deg}}\left({ }^{\circ}\right)$ is the change in the resonance angle before and after protein adsorption.

Table I. Physicochemical properties of the polymer brush layers.

\begin{tabular}{lcccc}
\hline Polymer brush layer & Poly(MPC) & Poly(TMAEMA) & Poly(SPMA) & Poly(BMA) \\
\hline Graft density (chains $/ \mathrm{nm}^{2}$ ) & 0.33 & 0.45 & 0.55 & 0.75 \\
Static contact angle of air in water $\left(^{\circ}\right)$ & $9 \pm 2$ & $17 \pm 0$ & $13 \pm 2$ & $73 \pm 3$ \\
$\zeta$-potential $(\mathrm{mV})$ & $-5.9 \pm 2.1$ & $64.9 \pm 3.6$ & $-74.0 \pm 11.7$ & $-37.2 \pm 6.1$ \\
\hline
\end{tabular}




\section{RESULTS AND DISCUSSION}

3.1 Well-characterized polymer brush surfaces

In this study, four polymer brush surfaces were utilized as model surfaces to systematically understand the relationship between the direct interaction force and the adsorption behavior of fibrinogen, namely, the zwitterionic poly(MPC), the cationic poly(TMAEMA), the anionic poly(SPMA), and the hydrophobic poly(BMA) brush surfaces. The physicochemical properties of these polymer brush surfaces are summarized in Table I. The graft densities (densities of the polymer chains per unit area) of all polymer brush layers were greater than 0.10 chains $/ \mathrm{nm}^{2}$. These values are high enough to form a highly dense polymer brush structure [11]. The static air contact angles on the polymer brush surfaces in water were below $20^{\circ}$ except for the poly(BMA) brush surface, which is bearing the hydrophobic groups in the side chains. This indicates that these polymer brush surfaces are hydrophilic in aqueous condition except for the poly(BMA) brush surface. The $\zeta$-potential of the polymer brush surfaces reflected the charge properties of the polymer chains. These demonstrate the fabrication of well-characterized polymer surfaces with controlled properties.

\subsection{Direct interaction force with fibrinogen}

Fig. 3 and Fig. 4 show the representative $f$ - $d$ curves and the strength of the direct interaction force between fibrinogen and the polymer brush surfaces in PBS, respectively. In the case of the zwitterionic poly(MPC) brush surface, the direct interaction force with fibrinogen was below $0.4 \mathrm{nN}$. That is, this surface hardly interacted with fibrinogen. On the other hand, strong direct interaction forces were detected on the cationic poly(TMAEMA), anionic poly(SPMA), and hydrophobic poly(BMA) brush surfaces (1.0- $6.0 \mathrm{nN})$.

\begin{tabular}{ccc}
\multicolumn{3}{l}{ Table II. Properties of fibrinogen. } \\
\hline Molecular weight & $\mathrm{p} I$ & Size \\
\hline $340 \mathrm{kDa}$ & 5.8 & $6.5 \times 6.5 \times 47.5 \mathrm{~nm}^{3}$ \\
\hline
\end{tabular}
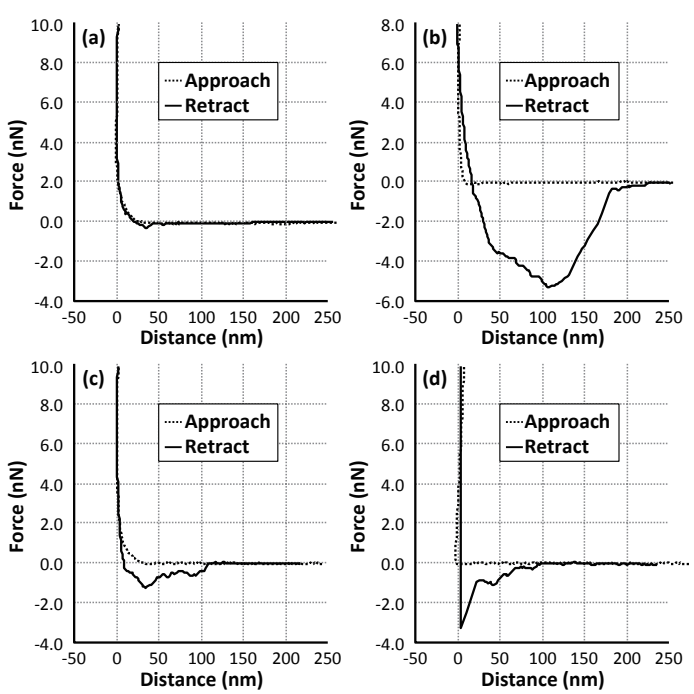

Fig. 3. Representative $f-d$ curves between fibrinogen and (a) poly(MPC), (b) poly(TMAEMA), (c) poly(SPMA), and (d) poly(BMA) brush surfaces in PBS.

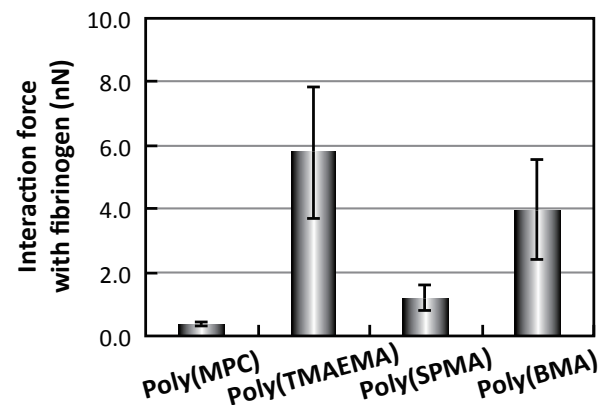

Fig. 4. Direct interaction force between fibrinogen and polymer brush surfaces in PBS.

In our previous study, the surface interaction forces generated on each polymer brush surface were analyzed by the $f$ - $d$ curve measurements of AFM using probes modified with the same polymer brush layer as the substrate [5]. Briefly, in the case of the cationic poly(TMAEMA) or the anionic poly(SPMA) brush surfaces, repulsive forces, which decreased depending on the ionic strength of the medium, were detected; these were considered to be electrostatic interaction forces. In the case of the hydrophobic poly(BMA) brush surface, while no repulsive or attractive force was detected during approach process, a strong adhesive force was detected during retraction process after contact of the two surfaces; this was considered to be hydrophobic interaction force. These forces were not detected on the zwitterionic poly(MPC) brush surface. That is, only electrostatic interaction was generated on the cationic poly(TMAEMA) brush surface and the anionic poly(SPMA) brush surface, and only hydrophobic interaction was generated on the hydrophobic poly(BMA) brush surface. Also, there was no such interaction force on the zwitterionic poly(MPC) brush surface. Therefore, electrostatic interaction and hydrophobic interaction were clearly separated on these systematically fabricated polymer brush surfaces.

That is, strong direct interaction forces with fibrinogen detected here would be originated from the only electrostatic interaction in the case of the poly(TMAEMA) brush surface and the poly(SPMA) brush surface, and from the only hydrophobic interaction in the case of the poly(BMA) brush surface. Fibrinogen is a rod-like protein with relatively high molecular weight, composed of negatively charged domains and positively charged domains [12]. Fibrinogen has its isoelectric point $(\mathrm{p} I)$ at 5.8 , and is net negatively charged as a whole at $\mathrm{pH} 7.4$, which would lead to the strongest interaction force between the cationic poly(TMAEMA) brush surface and fibrinogen via electrostatic interaction. On the other hand, fibrinogen actually has positively charged domains. This would result in the relatively strong interaction force with the anionic poly(SPMA) brush surface, though the force was not strong as with the poly(TMAEMA) or poly(BMA) brush surface. Also, the strong interaction with the hydrophobic poly(BMA) brush surface would be reasonable, considering that this surface interacted with proteins (bovine serum albumin, chicken egg white lysozyme) regardless of the charge properties in our previous study [5]. In contrast, in the case of the poly(MPC) brush surface, it is considered that fibrinogen could be easily detached from the surface 
even after contact of them, because this surface did not generate electrostatic or hydrophobic interaction.

3.3 Fibrinogen adsorption on polymer brush surfaces

Fig. 5 shows the amount of fibrinogen adsorbed on the polymer brush surfaces in PBS. Fibrinogen hardly adsorbed on the poly(MPC) brush surface $\left(<5.0 \mathrm{ng} / \mathrm{cm}^{2}\right)$. On the other hand, on the poly(TMAEMA), poly(SPMA), and poly(BMA) brush surfaces, which showed the strong direct interaction force with fibrinogen, the amount of adsorbed fibrinogen were approximately $750 \mathrm{ng} / \mathrm{cm}^{2}, 200 \mathrm{ng} / \mathrm{cm}^{2}$, and $400 \mathrm{ng} / \mathrm{cm}^{2}$, respectively. These results are consistent with those of our previous study, which showed that protein adsorption was dramatically suppressed on the poly(MPC) brush surface while a relatively large amount of proteins adsorbed on the other polymer brush surfaces [5].

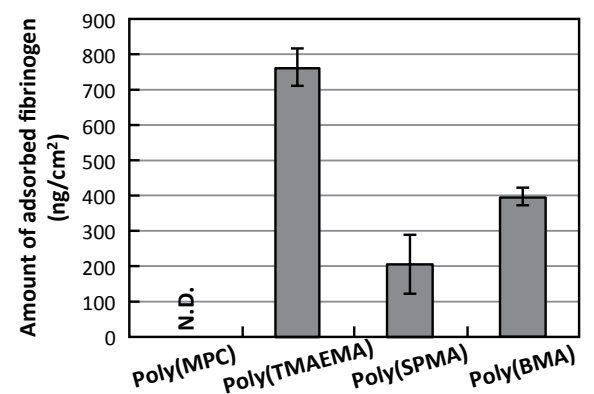

Fig. 5. Amount of fibrinogen adsorbed on the various polymer brush surfaces in PBS.

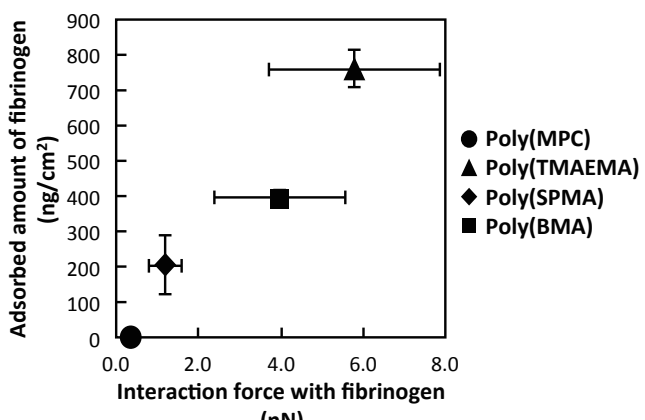

(nN)

Fig. 6. Relationship between the direct interaction force with fibrinogen and the amount of fibrinogen adsorbed on the various polymer brush surfaces.

There was a positive correlation between the direct interaction force with fibrinogen and the amount of adsorbed fibrinogen on the polymer brush surfaces (Fig. 6). Therefore, it was quantitatively indicated that the strength of the direct interaction force with fibrinogen would influence on the adsorption mass. Considering the protein adsorption process, at the stage in which fibrinogen repeatedly approach and depart from the surfaces reversibly, the spontaneous detachment of fibrinogen was inhibited on the surface which strongly interacted with fibrinogen. This would lead to the conformational change of fibrinogen, which strengthened the interaction between fibrinogen and the surfaces, and resulted in high adsorption mass of fibrinogen. The evaluation concerning the conformational change of fibrinogen adsorbed on the polymer brush surfaces is now under investigation. On the other hand, in the case of the zwitterionic poly(MPC) brush surface, fibrinogen could easily detach from the surface even when fibrinogen attached, because there was no significant interaction force. This led to a significant reduction of adsorption mass. Though the analysis focusing on the conformational change is important, the relationship was shown clearly as a preliminary study.

\section{CONCLUSIONS}

The relationship between the direct interaction force and adsorption behavior of fibrinogen were investigated using well-characterized polymer brush surface systems. Strong direct interaction forces with fibrinogen were detected on the cationic, anionic, and hydrophobic polymer brush surfaces after contact of fibrinogen and surfaces, which generated electrostatic interaction force or hydrophobic interaction force. Also, a large amount of fibrinogen adsorbed on these surfaces. On the other hand, the direct interaction force and adsorption of fibrinogen were dramatically suppressed on the zwitterionic polymer brush surface. We concluded that the design of surface exhibiting no interaction force would be important for non-biofouling materials.

\section{ACKNOWLEDGMENT}

This work was supported by a Grant-in-Aid for JSPS Fellow (15J10567) from Japan Society for the Promotion of Science.

\section{REFERENCES}

[1] H. Chen, L. Yuan, W. Song, Z. Wu, and D. Li, Prog. Polym. Sci., 33, 1059-1087 (2008)

[2] W.-B. Tsai, J. M. Grunkemeier, and T. A. Horbett, J. Biomed. Mater. Res., 44, 130-139 (1999)

[3] D. Leckband, and J. Israelachvili, Q. Rev. Biophys., 34, 105-267 (2001)

[4] Q. Wei, T. Becherer, S. Angioletti-Uberti, J. Dzubiella, C. Wischke, A. T. Neffe, A. Lendlein, M. Ballauff, and R. Haag, Angew. Chem., Int. Ed., 53, 8004-8031 (2014)

[5] S. Sakata, Y. Inoue, and K. Ishihara, Langmuir 31, 3108-3114 (2015)

bayashi, Polym. J., 22, 355-360 (1990)

[6] K. Ishihara, T. Ueda, and N. Nakabayashi, Polym. J., 22, 355-360 (1990)

[7] K. Matyjaszewski, P. J. Miller, N. Shukla, B. Immaraporn, A. Gelman, B. B. Luokala, T. M. Siclovan, G. Kickelbick, T. Vallant, H. Hoffmann, and T. Pakula, Macromolecules, 32, 8716-8724 (1999)

[8] Y. Inoue, T. Nakanishi, and K. Ishihara, React. Funct. Polym., 71, 350-355 (2011)

[9] D. M. Jones, A. A. Brown, W. T. S. Huck, Langmuir, 18, 1265-1269 (2002)

[10] I. Hirata, Y. Morimoto, Y. Murakami, H. Iwata, E. Kitano, H. Kitamura, and Y. Ikada, Colloids Surf., B, 18, 285-292 (2000)

[11] Y. Tsujii, K. Ohno, S. Yamamoto, A. Goto, and T. Fukuda, Adv. Polym. Sci., 197, 1-45 (2006)

[12] S.-Y. Jiang, S.-M. Lim, F. Albertorio, G. Kim, M. C. Gurau, R. D. Yang, M. A. Holden, and P. S. Cremer, J. Am. Chem. Soc., 125, 12782-12786 (2003)

(Received May 12, 2015; Accepted July 14, 2015) 